

\title{
Visual Contrast Sensitivity and Discrimination for 3D Meshes and their Applications
}

Georges Nader, Kai Wang, Franck Hétroy-Wheeler, Florent Dupont

\section{To cite this version:}

Georges Nader, Kai Wang, Franck Hétroy-Wheeler, Florent Dupont. Visual Contrast Sensitivity and Discrimination for 3D Meshes and their Applications. Computer Graphics Forum, 2016, 35 (7), pp.497-506. 10.1111/cgf.13046 . hal-01376275

\section{HAL Id: hal-01376275 \\ https://hal.inria.fr/hal-01376275}

Submitted on 9 Nov 2016

HAL is a multi-disciplinary open access archive for the deposit and dissemination of scientific research documents, whether they are published or not. The documents may come from teaching and research institutions in France or abroad, or from public or private research centers.
L'archive ouverte pluridisciplinaire HAL, est destinée au dépôt et à la diffusion de documents scientifiques de niveau recherche, publiés ou non, émanant des établissements d'enseignement et de recherche français ou étrangers, des laboratoires publics ou privés. 


\title{
Visual Contrast Sensitivity and Discrimination for 3D Meshes and their Applications
}

\author{
G. Nader ${ }^{1,2}$, K. Wang ${ }^{2}$, F. Hétroy-Wheeler ${ }^{3}$, F. Dupont ${ }^{1}$ \\ ${ }^{1}$ Université de Lyon, LIRIS UMR 5205 CNRS, France \\ ${ }^{2}$ CNRS, Univ. Grenoble Alpes, GIPSA-Lab, F-38000 Grenoble, France \\ ${ }^{3}$ Univ. Grenoble Alpes, LJK, F-38000 Grenoble, France and Inria
}

\begin{abstract}
In this paper, we first introduce an algorithm for estimating the visual contrast on a $3 D$ mesh. We then perform a series of psychophysical experiments to study the effects of contrast sensitivity and contrast discrimination of the human visual system for the task of differentiating between two contrasts on a 3D mesh. The results of these experiments allow us to propose a perceptual model that is able to predict whether a change in local contrast on 3D mesh, induced by a local geometric distortion, is visible or not. Finally, we illustrate the utility of the proposed perceptual model in a number of applications: we compute the Just Noticeable Distortion (JND) profile for smooth-shaded 3D meshes and use the model to guide mesh processing algorithms.
\end{abstract}

\section{Introduction}

Three-Dimensional (3D) meshes are nowadays more and more used in a large number of applications spanning over different fields such as digital entertainment, cultural heritage, scientific visualization, and medical imaging. In order to accommodate to the needs of these applications, it is common for 3D models to undergo various lossy operations (e.g., compression, simplification, watermarking) that introduce geometric artifacts to the model. This is a key issue since the introduced geometric artifacts might be visible on the rendered mesh. Although the importance of exploiting human perception has been recognized within the computer graphics community [CLL*13], most existing geometry processing algorithms are driven and/or evaluated by geometric metrics like Hausdorff distance [ASCE02] or root mean square error (RMS) [CRS98] which do not correlate with human perception [CLL*13]. Recently, a number of perceptually driven algorithms have been proposed. The goal of these methods is to evaluate the perceptual impact of geometric distortion or to guide geometric operations such as mesh compression and simplification. However, existing methods are usually based on assumptions about the general behavior of the human visual system instead of taking advantage of the characteristics of its internal mechanism. In most cases, the perceptual analysis of existing methods is carried out using geometric features such as surface curvature and surface roughness which are not necessarily perceptually relevant attributes. In fact, the human visual system is especially sensitive to variation in light energy, i.e., contrast, rather than its absolute magnitude [Wan95]. In particular, the analysis of contrast information has been the basis in many studies related to the analysis of visibility of a distortion introduced to a visual stimulus [Lin06, Da193]. In this paper, we present an algorithm for estimating contrast on a 3D mesh and propose a perceptual model that is based on an experimental study of the properties of the human visual system. In particular, we focus our study on the contrast sensitivity and contrast discrimination. This perceptual model can then be integrated into mesh processing algorithms in order to control the visual quality of the output.

The rest of this paper is organized as follows. Section 2 briefly reviews some related work. In Section 3 we introduce a method for estimating the contrast on smooth-shaded 3D meshes. In Section 4 we present a series of psychophysical experiments that were carried out in order to study the properties of the human visual system while observing a 3D mesh. The results of those experiments are then used to build a perceptual model that is able to predict whether a change in local contrast on the 3D mesh is visible or not. This perceptual model is later used in several mesh processing applications (Section 5). First we describe a method for computing the threshold beyond which the displacement of a vertex becomes visible (Section 5.1). Then we present perceptual methods to select the optimal vertex quantization level for a 3D mesh (Section 5.2) and to locally adapt the subdivision level in a 3D mesh (Section $5.3)$.

\section{Background}

This section is divided into two parts. The first part gives a quick overview about the major characteristics of the human visual system such as the contrast sensitivity and discrimination. A more detailed treatment of these characteristics can be found in the supplementary material or by referring to [Wan95]. In the second part, 
we present the related work in the field of perceptually adaptive computer graphics.

\subsection{Characteristics of the Human Visual System}

One important feature of the human visual system is that it is primarily sensitive to variation in light energy, rather than to its absolute value [Wan95]. This variation in light energy of a visual pattern is generally described by its contrast. The study of the properties of the human visual system relative to the perception of contrast forms the basis for nearly all visual perception [Wan95].

Contrast Sensitivity A visual pattern is visible to the human observer only if its contrast is above a certain threshold. This threshold is affected by the pattern's spatial frequency and by the global luminance of the observed scene. The spatial frequency is expressed in terms of cycles per degree (cpd) which corresponds to the number of patterns in one degree of the visual field. The reciprocal of the contrast visibility threshold is the contrast sensitivity. The contrast sensitivity function (CSF), introduced by Campbell and Robson [CR68], is a mathematical model that describes the evolution of the visibility threshold with respect to the spatial frequency and luminance level. It represents the visual system's band-pass filter characteristics when it comes to contrast sensitivity. In general, the sensitivity of the visual system peaks at around 2 to $5 \mathrm{cpd}$ then drops off to a point where no detail can be resolved [CR68]. At low luminance levels the contrast threshold increases when the average luminance decreases, while it becomes relatively stable for luminance levels above $100 \mathrm{~cd} / \mathrm{m}^{2}$ [Bar89].

Contrast Discrimination When the contrast of a visible visual pattern is altered, visual masking often affects the ability of the human visual system to detect this change in contrast. In other terms, the human visual system often has difficulties in discriminating between two similar patterns with different visible contrasts. The effects of visual masking are mainly caused by two factors: The initial contrast value and the regularity of the visual pattern.

- Effects of contrast value: The experiments of Legge and Foley [LF80] have shown that as the initial contrast of a visual pattern increases the threshold needed to detect a change in that contrast increases as well, approximately in a linear way.

- Effects of visual regularity: The theory of the free-energy principle [Fri10] suggests that the brain cannot fully process all of the visual information that it receives and tries to avoid surprises (i.e., information with uncertainties, which are usually from irregular visual patterns). This means that patterns with obvious statistical regularities are easier to be predicted and understood than those without any regularity. As a result, the change in information (i.e., contrast) in a regular visual pattern can be easily detected while it would be difficult to detect for an irregular one. This means that as the visual pattern becomes more irregular, the threshold for detecting a change in that pattern increases.

\subsection{Perception in Computer Graphics}

Over the past few years, the computer graphics community has recognized the importance of exploiting the limitations of the human visual system [CLL*13] as perceptually driven methods have proven to be useful for several applications. More specifically, perceptual methods have played an important role in estimating the visual quality of a distorted mesh [CLL*13], simplifying [LVJ05, SLMR14] and compressing [MVBH15] 3D models. Most of these methods are based on observations about the general behavior of the human visual system while observing 3D models. For example, perceptual metrics mostly rely on the idea that smooth surfaces are more sensitive to visual artifacts than rough ones [Lav09]. Existing perceptual methods also usually rely only on geometric attributes such as surface roughness [CDGEB07, WTM12], surface curvature [LVJ05, Lav11] and dihedral angles [VR12, MVBH15] to carry out the perceptual analysis rather than estimating perceptually relevant attributes such as contrast. Consequently, these methods are in general neither easily applicable to models of different properties (size, details and density) nor capable of adapting to varying circumstances of mesh usage (display characteristics, scene illumination and viewing distance).

Perceptual methods have also been extensively used throughout the image processing literature [LK11]. One of the most popular image-based techniques is Daly's Visual Difference Predictor (VDP) [Dal93] whose goal is to compute a map predicting the probability of detecting a distortion in the image. This approach has been used later to guide global illumination computations [RPG99]. However, the perceptual analysis in these methods is carried out on 2D rendered images and based on low-level properties of the human visual system that tend to overestimate the effects of visual artifacts in irregular regions. To overcome this limitation and still in the case of $2 \mathrm{D}$ images, several methods have recently tried to include the free-energy principle theory [WSL* $\left.13, \mathrm{DFL}^{*} 15\right]$ into the perceptual analysis which takes into account the irregularity of the visual pattern. Furthermore, there have been several attempts to use image-based methods for guiding geometry processing operations such as mesh simplification [QM08, MG10]. However, these methods remain limited as the rendered image of the detailed mesh model is needed. In addition, several studies [CHM*12, LLV16] have concluded that image-based techniques originated from the image/video processing communities might not be suited to evaluate the visual impact of a $3 \mathrm{D}$ geometric distortion as they introduce a different type of visual artifacts.

More recently, Nader et al. [NWHWD16] proposed an algorithm for computing the threshold beyond which the displacement of a vertex on a $3 \mathrm{D}$ mesh becomes visible. Their goal was to detect whether a distortion introduced to the mesh is visible or not. Their method is based on an experimental study of low-level properties of the human visual system such as the contrast sensitivity function and contrast masking. However, the proposed method works only for models illuminated by a directional light and rendered with a flat-shaded algorithm as a result of using a limited contrast estimation method. In addition, the perceptual model used for computing the visibility of the geometric distortions does not take into account the regularity of the visual pattern which results in overestimating the perceptual impact of distortions in complex regions of a $3 \mathrm{D}$ mesh.

In this paper, compared with [NWHWD16] considering only flat-shaded meshes with a directional light, we generalize the 


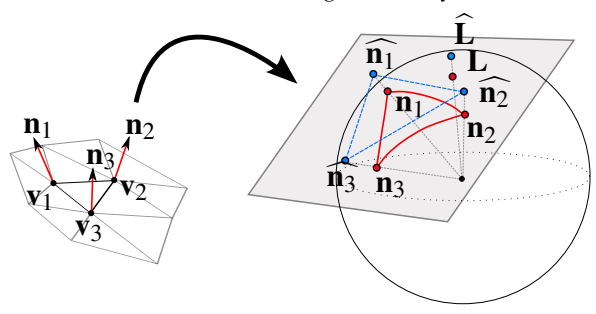

Figure 1: The projection of the normals, $\left[\mathbf{n}_{1}, \mathbf{n}_{2}, \mathbf{n}_{3}\right]$, on the unit sphere and to the tangent plane allows us to compute the barycentric coordinates of the closest and farthest points to the light direction $\mathbf{L}$.

method of estimating the contrast on a 3D model to smooth shading algorithms and different illumination types. Different from [NWHWD16], the CSF studied in this paper depends not only on the spatial frequency, but also on the scene's global luminance. Moreover, based on the free-energy principle, we propose a method to compute the visual regularity of a rendered mesh which allows us to take it into account while computing the visibility threshold. We showcase the utility of the proposed perceptual model by integrating it to a number of mesh processing applications such as selecting the optimal vertex quantization level of a mesh and locally adapting the level of subdivision in an adaptive mesh refinement setting.

\section{Contrast Estimation for Smooth-Shaded 3D Meshes}

As we mentioned earlier, the human visual system is primarily sensitive to variation in light energy, i.e., contrast [Wan95]. In digital images, the local contrast, $c$, attributed to a certain pixel is in general estimated using Michelson's definition:

$$
c=\frac{L_{\max }-L_{\min }}{L_{\max }+L_{\min }}
$$

where $L_{\max }$ and $L_{\min }$ correspond to the luminance values of the pixels with respectively the highest and lowest luminance in a predefined neighborhood. The goal of this study is to be able to compute the contrast on a 3D mesh that is rendered with a smooth shading algorithm. In that case, each point on a triangular face surface is attributed a luminance value. Therefore similarly to the imagebased definition of the local contrast, computing the contrast of a face requires finding the points corresponding to the highest and lowest luminance values. In this section, we propose an analytical method to compute these points. This will allow us to estimate the Michelson contrast for a given face. We limit our study to untextured diffuse surfaces. Let $F$ be a face of a 3D mesh determined by the vertices $\mathbf{v}_{1}, \mathbf{v}_{2}$ and $\mathbf{v}_{3}$ and let $\mathbf{x}_{i}$ be a point belonging to $F$. The surface normal at $\mathbf{x}_{i}$ is obtained using a barycentric interpolation of the corresponding vertex normals, $\mathbf{n}_{1}, \mathbf{n}_{2}$ and $\mathbf{n}_{3}$ :

$$
\mathbf{n}_{\mathbf{x}_{i}}=\frac{\mathbf{h}_{\mathbf{x}_{i}}}{\left\|\mathbf{h}_{\mathbf{x}_{i} \|}\right\|} \quad ; \quad \mathbf{h}_{\mathbf{x}_{i}}=N \times \mathbf{b}_{\mathbf{x}_{i}}
$$

where $N=\left[\mathbf{n}_{1}, \mathbf{n}_{2}, \mathbf{n}_{3}\right]$ is the matrix of vertex normals and $\mathbf{b}_{\mathbf{x}_{i}}=$ $\left[\alpha_{i}, \beta_{i}, 1-\alpha_{i}-\beta_{i}\right]^{T}$ is the vector of barycentric coordinates of $\mathbf{x}_{i}$. In the case of a diffuse surface, the luminance attributed to $\mathbf{x}_{i}$ is proportional to the cosine of the angle between the surface normal $\mathbf{n}_{\mathbf{x}_{i}}$ at $\mathbf{x}_{i}$ and the light direction $\mathbf{L}$. So finding the brightest and darkest points of a face boils down to finding the points with re-

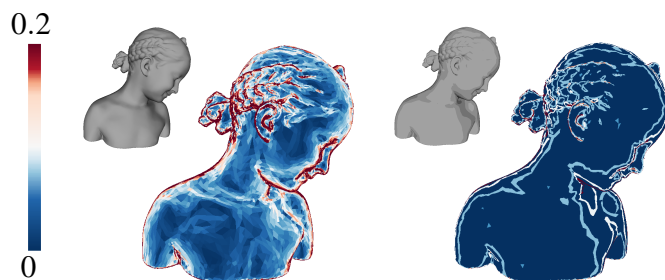

Figure 2: The Michelson contrast computed for each face of the Bimba model for a regular smooth shading algorithm (left) and cell shading rendering (right).

spectively the smallest and biggest angle between the corresponding normal and light direction. This can be achieved by computing their barycentric coordinates as explained below. We first map the normals of all the points $\mathbf{x}_{i} \in F$ and the light direction $\mathbf{L}$ onto the unit sphere (Fig. 1). It is easy to prove that the set of normals of $F$ forms a spherical triangle on the unit sphere as the normals of each edge of $F$ correspond to a geodesic on the unit sphere. Let

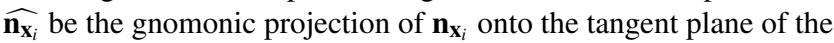
unit sphere at the centroid of the spherical triangle (Fig. 1) and let $\widehat{\mathbf{L}}$ be the projection of $\mathbf{L}$. The gnomonic projection is especially useful to our purposes since it projects geodesics to straight lines. In consequence, the points $\widehat{\mathbf{n}_{\mathbf{x}}}$ determine a euclidean triangle $\widehat{F}$ in the tangent plane. This means that finding the barycentric coordinates of the points with the smallest and biggest angles between the normal and light direction can be achieved by computing the barycentric coordinates of closest and farthest points between $\widehat{F}$ and $\widehat{\mathbf{L}}$. For $\mathbf{x}_{i} \in F$, the distance between corresponding $\widehat{\mathbf{n}_{\mathbf{x}_{i}}}$ and $\widehat{\mathbf{L}}$ can be expressed as:

$$
d_{\mathbf{x}_{i}}(\alpha, \beta)^{2}=\left\|\alpha \widehat{\mathbf{n}_{3} \mathbf{n}_{1}}+\beta \widehat{\mathbf{n}_{3} \mathbf{n}_{2}}+\widehat{\mathbf{L n}}\right\|^{2},
$$

where $\alpha, \beta$ are the barycentric coordinates of $\mathbf{x}_{i}$. The barycentric coordinates relative to the point with the highest and lowest luminance can finally be obtained by solving the following systems:

$$
\begin{array}{r}
\operatorname{argmin}\left\{d_{\mathbf{x}_{i}}(\alpha, \beta)\right\}, \quad \alpha+\beta \leq 1 \text { and } \alpha, \beta \in[0,1] ; \\
\operatorname{argmax}\left\{d_{\mathbf{x}_{i}}(\alpha, \beta)\right\}, \quad \alpha+\beta \leq 1 \text { and } \alpha, \beta \in[0,1] .
\end{array}
$$

The distance defined in Eq. (3) represents a paraboloid of parameters $\alpha$ and $\beta$. This makes possible to find its minimum and maximum using an efficient and analytical way. A detailed description of the solution of Eq. (4) can be found in the supplementary material. Having computed the brightest and darkest points of a face, it is now possible to evaluate its Michelson contrast. The contrast computed according to the method described above is compatible with directional light sources. It is also possible to extend this method to point light sources by assigning to each point $\mathbf{x}_{i} \in F$ a light direction according to:

$$
\mathbf{l}_{\mathbf{x}_{i}}=\frac{\mathbf{g}_{\mathbf{x}_{i}}}{\left\|\mathbf{g}_{\mathbf{x}_{i}}\right\|} \quad ; \quad \mathbf{g}_{\mathbf{x}_{i}}=\mathbf{x}_{i}-\mathbf{p}=M \times \mathbf{b}_{\mathbf{x}_{i}}-\mathbf{p}
$$

where $\mathbf{l}_{\mathbf{x}_{i}}$ is the light direction at $\mathbf{x}_{i}, \mathbf{p}$ is the light position, $M=$ $\left[\mathbf{v}_{1}, \mathbf{v}_{2}, \mathbf{v}_{3}\right]$ is the matrix of vertex position and $\mathbf{b}_{\mathbf{x}_{i}}$ is vector of barycentric coordinates of $\mathbf{x}_{i}$. For the same reason, the mapping of the light directions on the unit sphere will form a spherical triangle as the light directions assigned to edges of the face correspond to a geodesic and thus creating a euclidean triangle when projected to the tangent plane. Finally, The distance between $\widehat{\mathbf{n}_{\mathbf{x}}}$ and $\widehat{\mathbf{l}_{i}}$ on 



Figure 3: The spatial frequency is related to the size of the visual pattern $\left(d_{p h}\right)$, with respect to the size of one degree of the visual angle $\left(d_{1 \text { lcpd }}\right)$ on the display.

the tangent plane can be evaluated as:

$$
d_{\mathbf{x}_{i}}(\alpha, \beta)^{2}=\left\|\alpha\left(\widehat{\mathbf{n}_{3} \mathbf{n}_{1}}-\widehat{\mathbf{l}_{3} \mathbf{l}_{1}}\right)+\beta\left(\widehat{\mathbf{n}_{3} \mathbf{n}_{2}}-\widehat{\mathbf{l}_{3} \mathbf{l}_{2}}\right)+\left(\widehat{\mathbf{l}_{3} \mathbf{n}_{3}}\right)\right\|^{2} .
$$

By solving Eq. (4) for the distance in Eq. (6) we can evaluate the Michelson contrast for 3D models illuminated by a point light. The proposed method can be adapted to different illumination types and smooth shading algorithms. For example, Fig. 2 shows the contrast computed on a 3D mesh rendered with two different shadings. Notice how the contrast of the faces relative to the cell shaded rendering of the $3 \mathrm{D}$ model is 0 except for the ones where a transition in luminance occurs.

\section{Experimental Study and Threshold Model}

In this section we present our experimental study of the properties of the human visual system, which consists of measuring the threshold needed to detect the visual artifacts caused by a displacement of a vertex on the 3D mesh. In addition, we use the results of these experiments to compute the threshold beyond which a change in contrast becomes noticeable by a human observer.

\subsection{Contrast Sensitivity}

Contrast sensitivity refers to reciprocal of the threshold beyond which a visual signal becomes visible [Wan95]. The value of the visibility threshold is a function of the signal's spatial frequency and the global luminance of the observed scene which is estimated by multiplying the display's brightness by the light's energy. In order to measure the effects of contrast sensitivity on 3D models we first explain how the spatial frequency is computed.

Spatial Frequency The spatial frequency is related to the size of the visual pattern, with respect to the size of one degree of the visual angle (Fig. 3). It is expressed in terms of cycles per degree (cpd). So estimating the spatial frequency in the 3D setting requires first converting the size of the visual stimulus from its virtual value in the $3 \mathrm{D}$ world to its physical size on the display. To do so, we start by computing the virtual distance of the visual stimulus. It corresponds to the distance between the brightest and darkest points on a triangular face. These points can be obtained using the method described in Section 3. We then compute the number of pixels that the visual pattern occupies on the screen by applying a perspective projection. Having evaluated the number of pixels, the physical size of the visual pattern is then computed using the display's properties (resolution and size) as:

$$
d_{\mathrm{ph}}=\frac{n_{\mathrm{px}}}{\sqrt{r_{h}^{2}+r_{v}^{2}} / s},
$$



$2 \mathrm{cpd}$

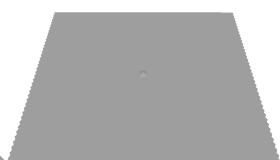

$8 \mathrm{cpd}$

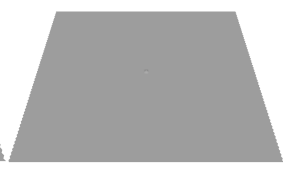

$16 \mathrm{cpd}$
Figure 4: Increasing the vertex density of the plane would increase the spatial frequency of the visual stimulus.

where $n_{\mathrm{px}}$ is the number of pixels of the displayed visual pattern, $r_{h}$ and $r_{v}$ are the display's horizontal and vertical resolution and $s$ its diagonal size. Finally the spatial frequency is estimated by:

$$
f=\frac{d_{1 \mathrm{cpd}}}{d_{\mathrm{ph}}}, d_{1 \mathrm{cpd}} \approx d_{\mathrm{eye}} \cdot \pi / 180,
$$

where $d_{1 \text { cpd }}$ is the size of one degree of the visual angle on the display and $d_{\text {eye }}$ is the distance between the eye and the display.

Experimental Protocol Two planes were displayed side by side on the screen, one of which exhibits a displaced vertex in its central area (Fig. 4). The displacement of the vertex causes a rotation in the normal direction of vertices belonging to the 1-ring neighborhood of the displaced vertex. As a consequence, the displacement of a vertex will cause a change in contrast of surrounding faces. The participants were then asked to answer by "Yes" or "No" to whether they can see any difference between the displayed planes, i.e., whether they detect the change in contrast caused by the vertex displacement. Following that answer, the magnitude of the vertex displacement is then adjusted according to the QUEST procedure [WP83], changing the value of the displayed contrast. This process is repeated until the threshold is found. In order to measure the threshold at different frequencies and global luminance levels, we respectively change the vertex density of the plane and the lighting conditions of the scene. The experiments took place in a low illuminated laboratory environment. The stimuli were displayed on a $1600 \times 120020$-inch monitor with a maximum luminance of $300 \mathrm{~cd} / \mathrm{m}^{2} .12$ subjects participated in our experiments. All had normal or corrected-to-normal vision and were 22 to 26 years old. No user interaction was allowed.

Results The threshold was measured for 7 spatial frequencies $(0.5,2,4,5.66,8,11.3$ and $16 \mathrm{cpd})$ and for 3 luminance levels $\left(180,110\right.$ and $\left.33 \mathrm{~cd} / \mathrm{m}^{2}\right)$. The results of these experiments are shown in Fig. 5. The plot shows a peak in sensitivity at around 3.5 cpd and a drop in sensitivity on either side of the peak. Additionally we can see that there is a decrease in sensitivity for low luminance while the sensitivity is relatively stable for a luminance level that is above $100 \mathrm{~cd} / \mathrm{m}^{2}$. The mean sensitivity over each frequency and luminance was then fitted to Barten's model [Bar89] that is defined by:

$$
\begin{aligned}
\operatorname{csf}(f, l) & =A(l) f e^{-B(l) f} \sqrt{1+c e^{B(l) f}}, \\
A(l) & =a_{0}(1+0.7 / l)^{a_{1}} \\
B(l) & =b_{0}(1+100 / l)^{b_{1}}
\end{aligned}
$$

with $a_{0}=125.42, a_{1}=0.09, b_{0}=0.343, b_{1}=0.17$ and $c=0.19$. While we have used a CSF mathematical model that is quite popular in the image/video processing communities, the sensitivity and peak frequency that we obtained have smaller values, for example 



Figure 5: Left: plot of the subjects' mean sensitivity over each frequency and luminance level. Right: plot of the 3D fit of Barten's CSF model [Bar89].

when compared with Daly's model [Da193]. This is in accordance with the observations of [CHM$\left.{ }^{*} 12\right]$ in which the authors' main objective is to test the efficiency of perceptual image-based techniques in the case of computer generated images. They concluded that image metrics are too sensitive for evaluating the visibility of computer graphics distortions. This is probably due to the difference in the types of visual artifacts caused by a geometric operation on the $3 \mathrm{D}$ mesh compared to the ones cause by an image processing operation. Meanwhile, it is interesting to note the impact the contrast sensitivity function has on the visibility of local geometric distortions on a 3D mesh. As the vertex density of a model increases from a low value, the 3D mesh becomes more sensitive to local geometric distortions since it becomes easier for the human visual system to detect them. However, if the density of the model passes a certain threshold, then it becomes hard to detect the local geometric distortions.

\subsection{Contrast Discrimination}

The ability of the human visual system to discriminate between two visible visual patterns depends on the initial contrast value and the pattern's visual regularity. Based on the free-energy principle, we first propose a method to estimate the regularity of a visual stimulus on a $3 \mathrm{D}$ mesh and then present the experiments that were carried out to measure the contrast discrimination threshold.

Visual Regularity The regularity of a visual pattern plays an important role in contrast discrimination. As we explained earlier, the brain can easily and successfully predict the visual patterns of a regular stimulus while irregular visual stimuli are difficult to be predicted [Fri10]. Based on this fact, we can relate the visual regularity to the prediction error of a visual pattern. We propose a computational model that aims to predict the contrast value of a triangular face based on the contrast information of its surrounding faces. The visual regularity can then be estimated from the residue between the actual contrast value and the predicted one. We suppose that the contrast of a triangular face $F$, denoted by $c$, can be estimated using a linear combination of the contrast of the three surrounding faces sharing an edge with $F$ :

$$
c^{\prime}=x_{1} c_{1}+x_{2} c_{2}+x_{3} c_{3},
$$

where $c^{\prime}$ is the estimated contrast and $c_{1}, c_{2}$ and $c_{3}$ are the contrast values of the adjacent faces organized in a descending order. So in order to evaluate $c^{\prime}$ we must estimate the linear coefficients
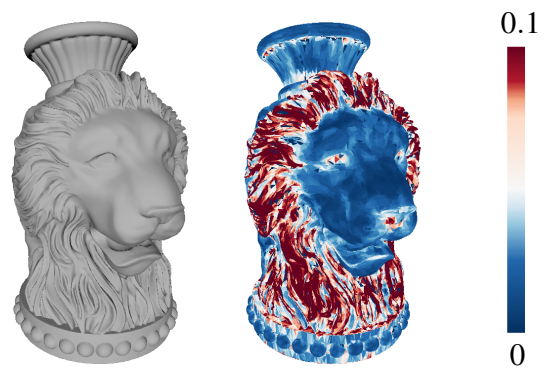

Figure 6: Visual regularity on the lion-vase model.



Figure 7: Visual stimuli for measuring the contrast discrimination threshold at different visual regularity levels.

$\left[x_{1}, x_{2}, x_{3}\right]$. This can be achieved by solving the following linear system using the least square regression method:

$$
\left[\begin{array}{ccc}
c_{1,1} & c_{1,2} & c_{1,3} \\
& \cdots & \\
c_{i, 1} & c_{i, 2} & c_{i, 3} \\
& \cdots & \\
c_{n, 1} & c_{n, 2} & c_{n, 3}
\end{array}\right]\left[\begin{array}{l}
x_{1} \\
x_{2} \\
x_{3}
\end{array}\right]=\left[\begin{array}{c}
c_{1} \\
\cdots \\
c_{i} \\
\cdots \\
c_{n}
\end{array}\right]
$$

where $c_{i}$ is the contrast value of the $i^{\text {th }}$ face within a predefined neighborhood centered at the current face $F, c_{i, 1}, c_{i, 2}, c_{i, 3}$ are the contrast values of the corresponding adjacent faces and $n$ is the total number of faces in the neighborhood. In practice we have used a neighborhood of a size equivalent to $3.5 \mathrm{cpd}$ which corresponds to the peak of fitted contrast sensitivity function (Section 4.1) in order to estimate the value of $\left[x_{1}, x_{2}, x_{3}\right]$ for each face. Finally the visual regularity (closer to 0 means more regular) assigned to a face $F$ is obtained by computing the absolute difference between the actual contrast and the estimated one:

$$
r=\left|c-c^{\prime}\right| \text {. }
$$

Figure 6 shows the visual regularity for the lion-vase model. Notice how the region containing the lion's mane is considered as visually irregular while the smooth face is visually regular.

Experimental Protocol Measuring the threshold beyond which a human observer is able to discriminate between two contrasts requires a visual stimulus that exhibits a visible initial contrast (i.e., above the CSF value) and certain visual regularity. We then gradually increase this initial contrast and measure the value needed to notice a change. In order to do so, we displace a series of vertices from a sphere approximated by a subdivided icosahedron. The icosahedron is subdivided 3 times which makes the contrast in each face visible for a human observer. By changing the light direction we can control the initial contrast value and by adding uniform noise to the sphere we can change its visual regularity (Fig. 7). We measure the discrimination threshold relative to 5 levels of visual 

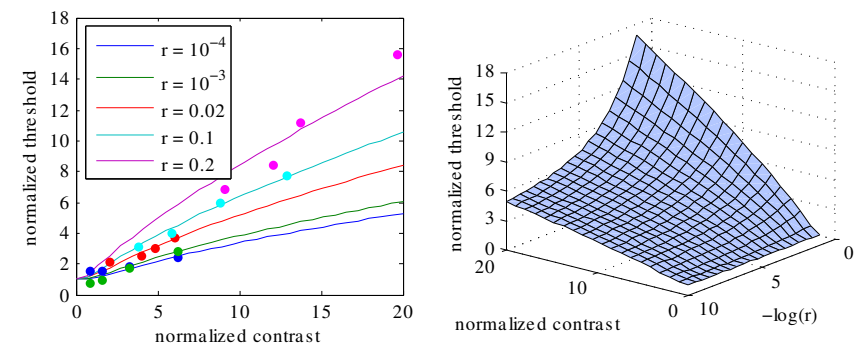

Figure 8: Left: plot of the subjects' mean threshold over each initial contrast value and visual regularity level. Right: plot of the $3 D$ fit of the contrast discrimination model (Eq. (13)).

regularity and 4 initial contrast values for each regularity level. The same experimental setup and method as in the contrast sensitivity experiments were adopted.

Results The results of these experiments are shown in Fig. 8. The plot shows the subjects' mean threshold for each of the visual regularity levels and initial contrast values. For the visible initial contrast whose normalized value is greater than 1 (normalization means multiplying by the corresponding CSF value), the measured threshold lies close to an asymptote with a slope increasing with the value of $r$. This means the less the human visual system is capable of predicting the observed surface, the higher the slope of the asymptote. This result is consistent with the analysis of Daly [Da193] which relates the value of the slope to the observer's familiarity with the observed stimulus. It also agrees with the observation that geometric distortions are more visible in smooth regions of the mesh than in rough ones [Lav09]. In order to take into consideration the visual regularity of a 3D mesh, we alter Daly's contrast discrimination model, $\operatorname{dis}(\tilde{c}, r)$, by mapping the value of visual regularity to the value of the slope using a Weibull function, $s(r)$ :

$$
\begin{aligned}
\operatorname{dis}(\tilde{c}, r) & =\left(1+\left(k_{1} \cdot\left(k_{2} \cdot \tilde{c}\right)^{s(r)}\right)^{b}\right)^{1 / b}, \\
s(r) & =(1-\delta)-(1-\gamma-\delta) \cdot e^{-10^{\beta(-\log (r)-\varepsilon)}}
\end{aligned}
$$

with $\tilde{c}$ the normalized contrast, $r$ the visual regularity and the fitted values $k_{1}=0.015, k_{2}=392.5, b=4, \gamma=0.63, \delta=-0.23, \beta=$ -0.12 and $\varepsilon=-3.5$.

\subsection{Contrast Threshold}

Having performed a series of psychophysical experiments whose goal was to study the effects of contrast sensitivity and discrimination in a 3D setting, we can now derive a computational model to evaluate the threshold $T$ beyond which a change in contrast becomes visible for the human observer. More precisely, to compute the threshold $T$, we divide the normalized threshold value obtained using Eq. (13) by the value of the CSF (Eq. (9)), as follows:

$$
T(c, f, l, r)=\frac{\operatorname{dis}(c \cdot \operatorname{csf}(f, l), r)}{\operatorname{csf}(f, l)},
$$

where $c$ is the original contrast, $r$ the corresponding contrast regularity, $f$ the spatial frequency, and $l$ the global luminance. The proposed threshold $T$ can adapt to various parameters. Firstly it takes into consideration the size and resolution of the display as well as the density of the mesh since they affect the frequency. Secondly it adjusts to the display's brightness and the light source's energy as they have an impact on the global luminance. And finally the threshold $T$ adjusts to the scene's illumination since it influences the initial contrast. Furthermore, for estimating the visibility probability of a change in contrast, it is common in the field of visual perception to use a psychometric function (Eq. (15)) with a slope set to 3.5 [MT86]:

$$
p(\Delta c)=1-e^{(\Delta c / T)^{3.5}}
$$

where $T$ is the contrast threshold and $\Delta c$ is the change in contrast.

\section{Applications}

In this section, we show how the proposed perceptual model can be useful for several mesh processing algorithms.

\subsection{Just Noticeable Distortion Profile}

The Just Noticeable Distortion (JND) profile refers to the threshold of a certain quantity beyond which the induced change in contrast becomes visible for the human observer. In the case of 3D meshes, it is evaluated by computing the maximum displacement each vertex can tolerate. To do so, we used an algorithm similar to the one proposed in [NWHWD16]. It can be summarized by the following: First we start by collecting all the faces that are affected by the displacement of a vertex. For each of these faces we estimate its contrast (Section 3), frequency (Section 4.1) and visual regularity (Section 4.2). This allows us to compute the threshold (Eq. (14)) beyond which a change in contrast for each face becomes visible. Then we gradually increase the displacement magnitude of the vertex and compute the change in contrast at each step. Using Eq. (15) we evaluate the probability of detecting that change in contrast. Once the detection probability reaches a certain value (e.g., 0.95 ), we attribute the displacement threshold to the corresponding displacement magnitude. It is important to note that the computed displacement threshold is relative to one light direction as the contrast value is affected by the scene's illumination. It is possible to compute the vertex displacement threshold independently from the light direction by evaluating the threshold according to multiple light directions sampled from a hemi-sphere around that vertex and then choosing the smallest one. A discussion about the practical implementation of the light independent threshold can be found in the supplementary material of [NWHWD16]. The light independent displacement threshold is particularly useful in an interactive scene. Some additional details regarding computing the vertex displacement threshold for an interactive scene can be found in the supplementary material of this paper.

In order to test the accuracy of the computed threshold and inspired by the literature on validation of image JND profiles, we have performed a subjective experiment where we injected noise into a number of 3D models with noise amplitude proportional to the computed threshold. The idea behind this experiment is to find the intensity from which the participants start noticing the injected noise in the model and compare it to the computed value. 
G. Nader, K. Wang, F. Hétroy-Wheeler, F. Dupont / Visual Contrast Sensitivity and Discrimination for 3D Meshes

\begin{tabular}{|c|l|l|l|l|l|}
\hline Models & Lion & Bimba & Horse & Dino & Venus \\
\hline Directional Light & 0.87 & 0.91 & 1.16 & 1.17 & 1.09 \\
Point Light & 0.81 & 0.89 & 0.97 & 1.11 & 1.05 \\
\hline
\end{tabular}

Table 1: Global noise energy value relative to JND modulated noise $\left(\beta_{\text {jnd }}\right)$.

Experimental Protocol The noise is injected into the 3D mesh according to:

$$
v_{i}^{\prime}=v_{i}+\mathrm{rnd} \cdot M\left(v_{i}\right) \cdot \mathbf{d i r}_{i},
$$

where $v_{i}$ is the $i^{\text {th }}$ vertex of the initial mesh and $v_{i}^{\prime}$ is the corresponding displaced vertex. dir $_{i}$ is the noise direction and rnd is a random value equal to either +1 or $-1 . M\left(v_{i}\right)$ represents the displacement magnitude, it is defined as:

$$
M\left(v_{i}\right)= \begin{cases}\beta_{\text {unif }} & \text { uniform noise, } \\ \beta_{\text {rough }} \cdot \operatorname{lr}\left(v_{i}\right) & \text { roughness modulated noise, } \\ \beta_{\text {jnd }} \cdot \operatorname{jnd}\left(v_{i}\right) & \text { JND modulated noise, }\end{cases}
$$

where $\beta_{\text {unif }}, \beta_{\text {rough }}$ and $\beta_{\text {jnd }}$ regulate the global noise intensity for each of the noise injection methods. $\operatorname{lr}\left(v_{i}\right)$ is the local surface roughness as defined in [WTM12] and jnd $\left(v_{i}\right)$ is the threshold value computed as explained above. Having three different energy modulations ( $\beta_{\text {unif }}, \beta_{\text {rough }}$ and $\beta_{\text {jnd }}$ ) allows us to compare the maximum amount of invisible noise each method can inject. This is particularly important as it indicates whether the noise injection method is able to locally adapt to the meshes' properties (density, size). Moreover we expect the measured $\beta_{j n d}$ to have a value close to 1 , since according to our method, if $\beta_{j n d}>1$ the random noise injected into the $3 \mathrm{D}$ mesh is expected to be visible otherwise it should remain invisible. We have adopted the same experimental protocol that we have used to measure the visibility thresholds. Two models were displayed on a $1600 \times 120020$-inch monitor, one of which has noise injected. The subjects had to answer by either "yes" or "no" whether they think the noise is visible. The intensity of the noise ( $\beta_{\text {unif }}, \beta_{\text {rough }}$ and $\beta_{\text {jnd }}$ ) is then adaptively adjusted according to the QUEST procedure [WP83]. The subjects were allowed to interact with the displayed models by rotating the camera around the model. 12 new subjects participated in the experiment. The experiment was carried out with two lighting conditions: front directional light and front point light whose energy decreases proportionally to the square of the distance to the model.

Results Table 1 and Fig. 9 show the results of this subjective experiment (some complementary results can be found in the supplementary material). We see that the measured $\beta_{\text {jnd }}$ is indeed close to 1 for all of the models, meaning that the proposed perceptual model is able to accurately compute the vertex displacement threshold. Additionally, plots (a) and (b) in Fig. 9 show the MRMS (maximum root mean square error [CRS98]) value of each model for the three noise intensity types. In all cases, the JND modulated models have the highest MRMS value indicating that our perceptual model is able to inject the highest amount of tolerable noise into the meshes. In addition, we point out that the models illuminated with the low energy point light can tolerate more noise than the ones illuminated with the high energy directional one since the reduced light energy effects the global luminance of the scene and thus reduces the sensitivity to contrast.
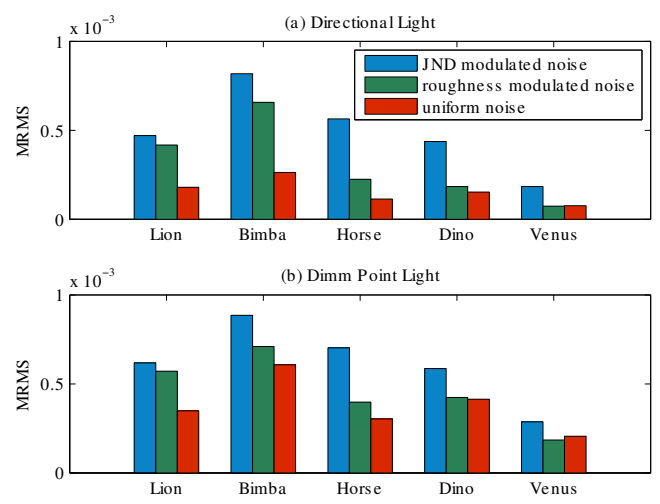

Figure 9: Plot of the MRMS induced by noise injection for three different types of noise at the same visibility level.
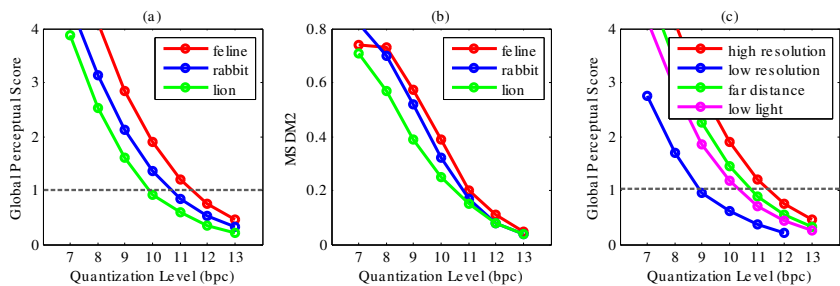

Figure 10: (a) The perceptual score versus the quantization levels (in bpc) of three models. (b) The MSDM2 [Lav11] score versus the quantization levels of three models. (c) Effects of the model resolution, object distance and light energy on the perceptual score.

The JND profile can be a useful tool when developing mesh processing methods. As demonstrated in our subjective validation, the proposed perceptual model can effectively guide the injection of vertex noise into the 3D mesh. This can have a direct application in the case of $3 \mathrm{D}$ watermarking and geometric compression algorithms (e.g., vertex coordinates quantization, see Section 5.2) as their corresponding performance usually relies on the degree of tolerable change in vertex coordinates. Moreover, in the supplementary material, we showcase how the vertex displacement threshold can be integrated into a perceptual mesh simplification process.

\subsection{Automatic Optimal Vertex Quantization Level Selection}

Similarly to [NWHWD16], we showcase the advantages that the proposed perceptual analysis can have in geometric operations such as vertex coordinates quantization. Vertex quantization in an essential step in most 3D mesh compression techniques. This operation introduces geometric distortions onto the 3D mesh that might be visible to a human observer. We use the vertex displacement threshold to compare the distorted mesh with its original version and this allows us to automatically select the optimal vertex quantization level (in bits per coordinate, bpc), i.e., the level with the highest quantization noise energy that is not visible. To do so, we start by computing a local visibility map which indicates whether the displacement of a vertex caused by the coordinates quantization is visible or not. The visibility of a vertex displacement is evaluated as the ratio between the magnitude of the displacement and the ver- 


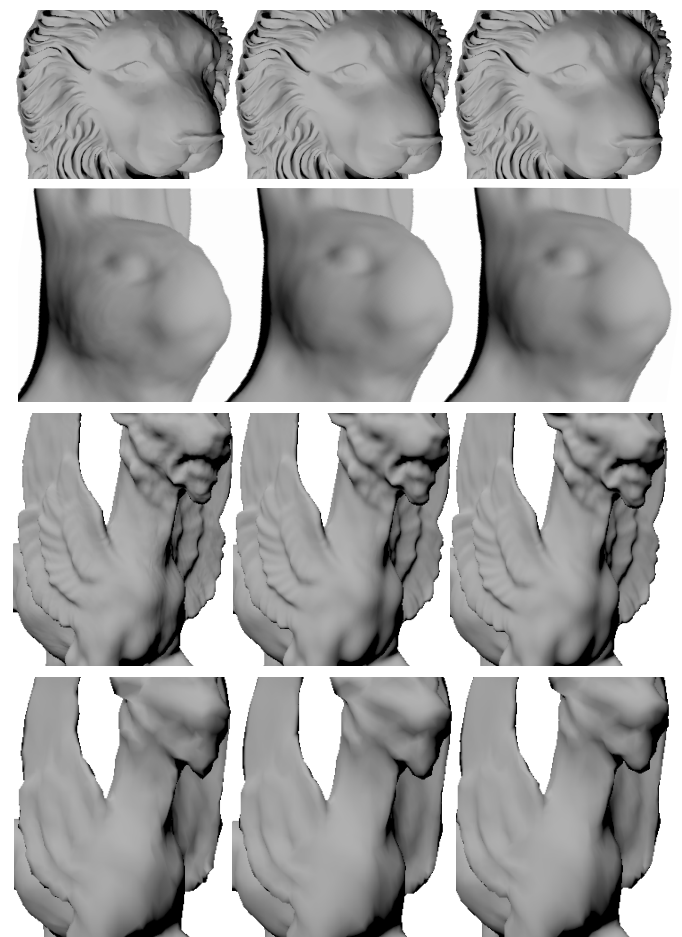

Figure 11: Quantized meshes with different quantization levels. The middle column corresponds to the optimal quantization level (10, 11, 12 and 9 bpc for respectively Lion, Rabbit, high-resolution Feline and low-resolution Feline). The right mesh corresponds to a one bit higher than the optimal level while the one on left corresponds to a one bit lower. (For better comparison between the models please refer to the electronic version of this paper.)

tex displacement threshold as follows:

$$
\vartheta_{i}=\frac{\left\|\mathbf{v}_{i}^{\prime}-\mathbf{v}_{i}\right\|}{\operatorname{jnd}\left(\mathbf{v}_{i}\right)}
$$

where $\mathbf{v}_{i}^{\prime}$ and $\mathbf{v}_{i}$ are the $i^{t h}$ vertices of respectively the distorted and the original mesh and jnd $\left(\mathbf{v}_{i}\right)$ is the vertex displacement threshold of $\mathbf{v}_{i}$ in the direction of $\mathbf{v}_{i}^{\prime}-\mathbf{v}_{i}$. Finally, we aggregate the local visibility values into a global visibility score using a Minkowski pooling technique as:

$$
S=\left(\frac{1}{n} \sum_{i=1}^{n} \vartheta_{i}^{p}\right)^{(1 / p)},
$$

where $n$ is the number of vertices in the mesh and $p=2$ is the Minkowski power. This score allows us to test whether the distortion introduced by the vertex quantization operation is globally visible. If $S \leq 1$, the noise magnitude is globally below the visibility threshold, which means that the distortion is not visible. On the other hand if $S>1$, the distortion becomes visible as the noise magnitude is in general above the visibility threshold. The optimal quantization level would be the lowest one where the global visibility score is below 1 . Figure 10 shows the global perceptual score versus the level of coordinates quantization for three meshes. According to the defined score the optimal quantization level is $10 \mathrm{bpc}$ for the Lion model, 11 for the Rabbit model and 12 for the Feline
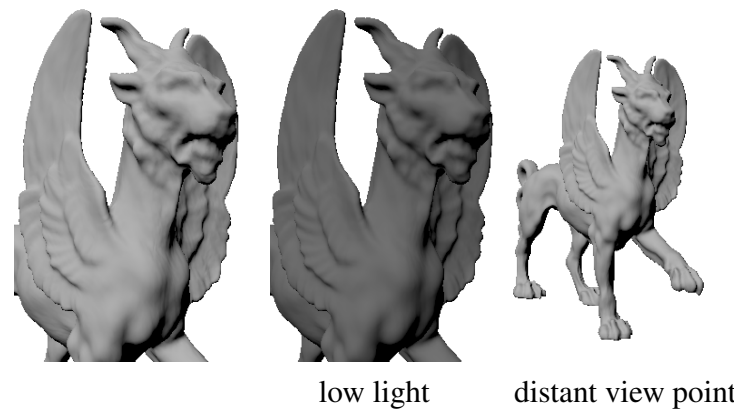

Figure 12: The high-resolution Feline model with an 11 bpc quantization level under different circumstances.

model. In addition, the proposed global perceptual score can adapt to different circumstances of mesh usage such as view distance, light energy and mesh resolution (Fig. 10 (c)). By reducing the resolution of the Feline model, the optimal quantization level goes down from $12 \mathrm{bpc}$ to $9 \mathrm{bpc}$ while a distant view or low energy light makes the optimal quantization level become $11 \mathrm{bpc}$. These results are consistent with the human observations as shown in Fig. 11 and Fig. 12. By contrary, we cannot obtain these results by thresholding the output of state-of-the-art mesh perceptual metrics such as [Lav11] (Fig. 10 (b)). In particular, the metric's output remains the same under different light energies and viewing distances.

\subsection{Perceptually Adaptive Mesh Subdivision}

Not only the JND profile, but also the proposed perceptual models, e.g., the CSF model (Eq. (9)), can be easily integrated to mesh processing algorithms. In an adaptive mesh refinement setting, the subdivision operation is applied to the faces that fulfill a certain condition. The subdivision is often applied to the faces that are relatively close to the viewpoint or are part of the displayed contour and the subdivision process is usually halted when a certain polygon budget is reached. In general, the goal of adaptive mesh refinement methods is to display a coarse model in a way that appears visually smooth which can be achieved if the normal vectors used for the shading computation produce a smooth visual pattern. In other words, we may consider that visual smoothness is achieved if the interpolation between the brightest and darkest luminance level inside a face is unnoticeable to a human observer. As a result, we can use the proposed perceptual model in order to test whether this interpolation is visible or not. This test can therefore be done by simply normalizing the contrast value by the CSF value:

$$
\tilde{c}=c \cdot \operatorname{csf}(f, l),
$$

where $\tilde{c}$ is the normalized contrast, $c$ is the face's contrast value, $f$ is the corresponding frequency and $l$ is the global luminance. Hence, the contrast is visible if its normalized value, $\tilde{c}$, is greater than 1 , otherwise it is not visible. Finally, the subdivision operation is applied to the faces whose contrast is visible. The subdivision operation is stopped once all the faces have an invisible contrast. Since the normalized contrast, $\tilde{c}$, takes into account the spatial frequency, then the proposed subdivision process will automatically adapt to the distance between the 3D model and the viewpoint.

We have tested this perceptual subdivision criterion using 


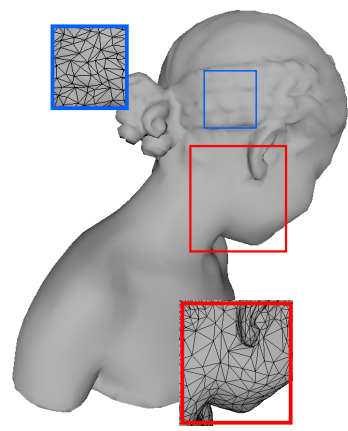

original $-3.5 \mathrm{k}$

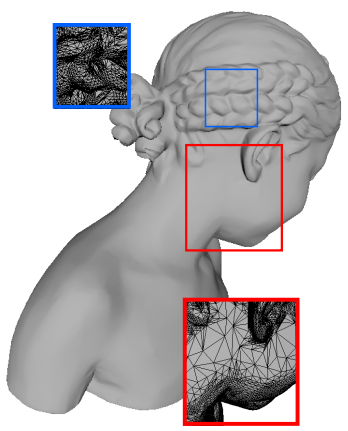

(a) $30 \mathrm{k}$ - ours

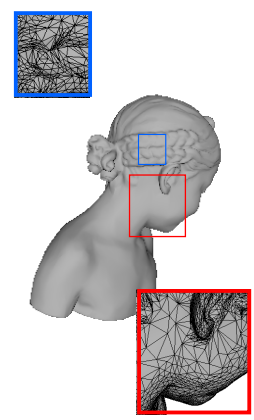

(b) $10 \mathrm{k}$ - ours

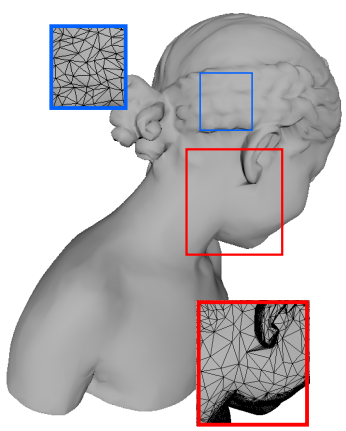

(c) $30 \mathrm{k}-[\mathrm{BA} 08]$

Figure 13: (a) The perceptual subdivision process converges at around 30k vertices and the resulting mesh appears to be visually smooth. (b) Increasing the viewing distance alters the spatial frequency and fewer subdivisions are required to obtain a visually smooth model. (c) The output model using the adaptive subdivision method in [BA08] at the same number of vertices as (a). The subdivisions are concentrated in the contour region leaving the rough part of the model intact.

Boubekeur and Alexa's [BA08] tessellation framework. Figure 13 shows a coarse version of the Bimba model that was subdivided using the proposed perceptually driven method. The subdivided version exhibits an increase in density in rough regions which usually contain faces with visible contrast. The subdivision process converges around 30k vertices and the resulting mesh (Fig. 13 (a)) appears to be visually smooth. By increasing the view distance, the spatial frequency of the observed visual stimuli is increased, thus reducing the sensitivity to contrast. This means that fewer subdivisions are needed in order to obtain a visually smooth model (Fig. 13 (b)). In addition, we have compared our perceptual subdivision criterion to the one proposed in [BA08] which consists in subdividing the faces that are part of the mesh's contour (Fig. 13 (c)). We stopped the subdivision process once the same number of vertices is attained. As expected the density of the resulting model was high in contour region while the rough part of the model remained untouched.

\section{Discussion and Limitations}

The method presented in this paper extends the previous work [NWHWD16], which was restricted to flat-shaded mesh illuminated with a directional light, to models rendered with a smooth shading algorithm. Moreover, our method is more flexible as it can adapt to directional and point light sources. Furthermore, we expand the perceptual model used in [NWHWD16] which is limited to the effects of spatial frequency to take into consideration the effects of global luminance and visual regularity on the visibility threshold. As a consequence, the computed visibility threshold can adapt to various mesh properties such as size and density, as well as to scene illumination. We have also presented a new application of adaptive mesh subdivision in which the perceptual model is used to judge whether the subdivision operation should be performed on a certain face or not. Finally, the visibility threshold can adjust to the resolution, size and luminance of the display device which are passed as parameters to the perceptual model. In our implementation, computing the vertex displacement threshold (i.e., the JND profile) for a model of approximately $100 \mathrm{k}$ vertices takes about $45 \mathrm{~s}$ while the subdivision of the Bimba model from $3.5 \mathrm{k}$ to $30 \mathrm{k}$ vertices takes only 9ms. A more detailed comparison with [NWHWD16] and discussion about the execution time can be found in the supplementary material.

The proposed perceptual model can be useful for geometric processing operations as it can help control the visual quality of the output or define criterion in order to guide the geometric algorithm. However, it has its own limitations. The proposed model does not take into account the color attributed to the mesh or the illumination as it focuses on white lumination levels. An accurate treatment of color would require conducting psychophysical experiments to measure the contrast threshold for each color channel as the sensitivity is different for various light frequencies [Kel83]. We can then apply the same perceptual analysis described in this paper for each channel in order to compute the visibility threshold. Another limitation is that the proposed perceptual method works for untextured diffuse surfaces that are illuminated by one light source and is restricted to local geometric distortions. This limitation is due to the algorithm used to compute the contrast on a triangular face. It means that the proposed method for computing the visibility threshold remains valid as long as this limitation can be addressed by extending the method for computing the contrast. Taking into account more advanced lighting such as environment maps, complex surface materials and large-scale geometric distortions would require a more general definition of contrast that could be based on a thorough and non-trivial analysis of the rendering algorithm without being restricted to a triangular face. Textures on the other hand can be taken into account by combining the contrast due to the shading with the contrast of the texture. Finally, even if the simple linear system used to estimate the visual regularity works well in practice, it would be interesting to test whether higher-order methods would give better results.

\section{Conclusion and Future Work}

In this paper, we have presented an analytical method for computing the contrast on a 3D triangular mesh. We then performed an experimental study of the properties of the human visual system while observing a 3D mesh in order to accurately measure the threshold beyond which a change in contrast becomes visible. The results of these experiments allow us to derive a computational model that evaluates the threshold beyond which a change in contrast of a tri- 
angular face becomes visible. This model can adapt to the various display parameters (resolution, size and brightness), to the size and density of the triangular mesh as well as to directional and point light illumination. We then illustrate the utility of the perceptual model in several applications: we compute the JND profile for smooth-shaded meshes, and we use the model to guide mesh processing algorithms including vertex coordinates quantization, mesh simplification (in supplementary material) and adaptive mesh subdivision.

We think that it would be possible, in a future work, to generalize the contrast analysis in order to compute a contrast value at different scales and for different orientations. This would allow us to take into account the characteristics of the human visual system that are related to the orientation and scale selectivity. it would also be interesting to exploit the utility of the proposed contrast algorithm in order to define high-level visual tasks such as mesh saliency. Finally, It would be useful to incorporate to the proposed perceptual model the dynamic aspect of the contrast sensitivity function. This would extend the perceptual model to dynamic meshes.

\section{Acknowledgments}

We would like to thank all the subjects who participated in the subjective experiments. This work is supported by the ARC6 program of the "Région Rhône-Alpes" through the PADME project.

\section{References}

[ASCE02] Aspert N., SANTA-CruZ D., Ebrahimi T.: MESH: Measuring error between surfaces using the Hausdorff distance. In Proc. of IEEE International Conference on Multimedia \& Expo (2002), pp. 705708. 1

[BA08] Boubekeur T., Alexa M.: Phong tessellation. ACM Transactions of Graphics 27, 5 (2008), 141. 9

[Bar89] BARTEN P. G. J.: The Square Root Integral (SQRI): A new metric to describe the effect of various display parameters on perceived image quality. In Proc. of SPIE (1989), vol. 1077, pp. 73-82. 2, 4, 5

[CDGEB07] Corsini M., Drelie Gelasca E., Ebrahimi T., Barni M.: Watermarked 3D mesh quality assessment. IEEE Transactions on Multimedia 9, 2 (2007), 247-256. 2

[CHM*12] CadíK M., Herzog R., Mantiuk R., Myszkowski K., SEIDEL H.-P.: New measurements reveal weaknesses of image quality metrics in evaluating graphics artifacts. ACM Transactions on Graphics 31,6 (2012), 1-10. 2, 5

[ClL*13] Corsini M., Larabi M.-C., Lavoué G., Petří́ O., VÁŠA L., WANG K.: Perceptual metrics for static and dynamic triangle meshes. Computer Graphics Forum 32, 1 (2013), 101-125. 1, 2

[CR68] CAMPBELl F. W., RobSON J.: Application of Fourier analysis to the visibility of gratings. The Journal of Physiology 197, 3 (1968), 551-556. 2

[CRS98] Cignoni P., Rocchini C., Scopigno R.: Metro: Measuring error on simplified surfaces. Computer Graphics Forum 17, 2 (1998), $167-174.1,7$

[Da193] DALY S.: The visible differences predictor: An algorithm for the assessment of image fidelity. In Digital Images and Human Vision, Watson A. B., (Ed.). MIT Press, Cambridge, MA, USA, 1993, pp. 179206. $1,2,5,6$

[DFL*15] Dong L., FAng Y., Lin W., Deng C., Zhu C., SeAH H. S. Exploiting entropy masking in perceptual graphic rendering. Signal Processing: Image Communication 33 (2015), 1-13. 2
[Fri10] FRISTON K.: The free-energy principle: a unified brain theory? Nature Reviews Neuroscience 11, 2 (2010), 127-138. 2, 5

[Kel83] Kelly D. H.: Spatiotemporal variation of chromatic and achromatic contrast thresholds. Journal of Optical Society of America 73, 6 (1983), 742-750. 9

[Lav09] LAvouÉ G.: A local roughness measure for 3D meshes and its application to visual masking. ACM Transactions on Applied Perception 5, 4 (2009), 1-23. 2, 6

[Lav11] LAVouÉ G.: A multiscale metric for 3D mesh visual quality assessment. Computer Graphics Forum 30, 5 (2011), 1427-1437. 2, 7,

[LF80] LEGGe G. E., Foley J. M.: Contrast masking in human vision. Journal of Optical Society of America 70, 12 (1980), 1458-1471. 2

[Lin06] LIN W.: Computational models for just-noticeable difference. In Digital Video Image Quality and Perceptual Coding, Wu H. R., Rao K. R., (Eds.). CRC Press, London, UK, 2006, pp. 281-303. 1

[LK11] LiN W., Kuo C.-C. J.: Perceptual visual quality metrics: A survey. Journal of Visual Communication and Image Representation 22, 4 (2011), 297-312. 2

[LLV16] Lavoué G., LaRabi M.-C., Vasa L.: On the efficiency of image metrics for evaluating the visual quality of $3 \mathrm{D}$ models. IEEE Transactions on Visualization and Computer Graphics (2016). doi: 10.1109/TVCG.2015.2480079. 2

[LVJ05] Lee C. H., Varshney A., Jacobs D. W.: Mesh saliency. ACM Transactions on Graphics 24, 3 (2005), 659-666. 2

[MG10] Menzel N., Guthe M.: Towards perceptual simplification of models with arbitrary materials. Computer Graphics Forum 29, 7 (2010), 2261-2270. 2

[MT86] MAYER M. J., TYLER C. W.: Invariance of the slope of the psychometric function with spatial summation. Journal of Optical Society of America A 3, 8 (1986), 1166-1172. 6

[MVBH15] Marras S., VÁša L., Brunnett G., Hormann K.: Perception-driven adaptive compression of static triangle meshes. Computer-Aided Design 58 (2015), 24-33. 2

[NWHWD16] Nader G., Wang K., Hétroy-Wheeler F., Dupont F.: Just noticeable distortion profile for flat-shaded 3D mesh surfaces IEEE Transactions on Visualization and Computer Graphics (2016). doi:10.1109/TVCG.2015.2507578.2,6, 7,9

[QM08] QU L., MeYer G. W.: Perceptually guided polygon reduction. IEEE Transactions on Visualization and Computer Graphics 14, 5 (2008), 1015-1029. 2

[RPG99] Ramasubramanian M., Pattanaik S., Greenberg D.: A perceptually based physical error metric for realistic image synthesis. In Proc. of ACM SIGGRAPH (1999), pp. 73-82. 2

[SLMR14] Song R., LiU Y., Martin R. R., Rosin P. L.: Mesh saliency via spectral processing. ACM Transactions on Graphics 33, 1 (2014), 1-17. 2

[VR12] VÁŠA L., RUS J.: Dihedral angle mesh error: a fast perception correlated distortion measure for fixed connectivity triangle meshes. Computer Graphics Forum 31, 5 (2012), 1715-1724. 2

[Wan95] Wandell B. A.: Foundations of Vision. Sinauer Associates, Sunderland, MA, USA, 1995. 1, 2, 3, 4

[WP83] Watson A. B., Pelli D. G.: QUEST: a Bayesian adaptive psychometric method. Perception \& Psychophysics 33, 2 (1983), 113120. 4,7

[WSL*13] Wu J., SHI G., LIN W., LIU A., QI F.: Just noticeable difference estimation for images with free-energy principle. IEEE Transactions on Multimedia 15, 7 (2013), 1705-1710. 2

[WTM12] Wang K., Torkhani F., Montanvert A.: A fast roughness-based approach to the assessment of 3D mesh visual quality. Computers \& Graphics 36, 7 (2012), 808-818. 2, 7 